\title{
Ameliorative effects of parecoxib in combination with ultrasound-guided paravertebral block (UGPB) on stress and inflammatory responses following thoracoscopic surgery
}

\author{
Xuejie $\mathrm{Li}^{1,2}$, Xiaoning $\mathrm{Cui}^{2}$, Shouhong Zhang ${ }^{2}$, Zhijian $\mathrm{Fu}^{1 *}$ \\ ${ }^{1}$ Shandong Provincial Hospital Affiliated to Shandong University, Jinan 250021, ${ }^{2}$ Department of Anesthesiology, Yantaishan \\ Hospital, Yantai 264000, PR China \\ ${ }^{\star}$ For correspondence: Email: zhijian_fu@163.com Tel/Fax: +86-0531-68776580
}

Sent for review: 3 February 2018

Revised accepted: 21 May 2018

\begin{abstract}
Purpose: To investigate the ameliorative effects of parecoxib combined with ultrasound-guided paravertebral block (UGPB) on stress and inflammatory responses after thoracoscopic surgery. Methods: Forty thoracoscopic surgery patients were randomized into placebo (control) and parecoxib groups. Parecoxib was administered pre-operation, $24 \mathrm{~h}$ and $48 \mathrm{~h}$ after operation. Arterial blood was collected, and endotoxin (ET), thromboxane A2 (TXA2), interleukin 6 (IL-6) and tumor necrosis factor alpha (TNF- $\alpha$ ) levels were measured. Opioid dosage, infusion volume, blood loss, operation time, visual analogue scale (VAS) score at $24 \mathrm{~h}$ and $48 \mathrm{~h}$, and hospitalization period were recorded.

Results: No significant differences were observed in age, sex, height, body weight, opioid dosage, surgery time, blood loss, or infusion volume between groups. VAS scores in the parecoxib group were significantly lower than the control group after 24 and $48 \mathrm{~h}$. The hospitalization period of the parecoxib group was significantly shorter than the control group. Plasma levels of ET, TXA2, IL-6 and TNF- $\alpha$ in the parecoxib group were lower than the control group after $24 \mathrm{~h}$; however, there was no significant difference after $48 \mathrm{~h}$.

Conclusion: Parecoxib, combined with UGPB, effectively relieves thoracoscopic pain, stress, and inflammatory responses of patients after thoracoscopic surgery. This treatment would improve the postoperative quality of life of lung cancer patients.
\end{abstract}

Keywords: Parecoxib, Paravertebral block, Stress response, Inflammatory response

\begin{abstract}
This is an Open Access article that uses a funding model which does not charge readers or their institutions for access and distributed under the terms of the Creative Commons Attribution License (http://creativecommons.org/licenses/by/4.0) and the Budapest Open Access Initiative (http://www.budapestopenaccessinitiative.org/read), which permit unrestricted use, distribution, and reproduction in any medium, provided the original work is properly credited.

Tropical Journal of Pharmaceutical Research is indexed by Science Citation Index (SciSearch), Scopus, International Pharmaceutical Abstract, Chemical Abstracts, Embase, Index Copernicus, EBSCO, African Index Medicus, JournalSeek, Journal Citation Reports/Science Edition, Directory of Open Access Journals (DOAJ), African Journal Online, Bioline International, Open-J-Gate and Pharmacy Abstracts
\end{abstract}

\section{INTRODUCTION}

Lung cancer is one of common malignant cancers in people over the age of 40 , and it remains the leading cause of cancer related death worldwide [1]. Although the current diagnosis of lung cancers is greatly improved, the 5-year survival rate of lung cancer is still only $70 \%$ [2]. Surgical resection is the most effective available therapy for lung cancer. However, after surgical resection, thoracic post-operative pain, stress response and inflammatory response 
seriously affect the sputum excretion and deep breathing of patients [3].

The pathological mechanisms of postoperative pain are complex, and inflammatory pain is one important aspect of postoperative pain [4]. Many peri-operative pro-inflammatory cytokines can lead to excessive stress response and disrupt the balance of cytokines [5]. Subsequently, the inflammatory response cascades is activated, leading to systemic inflammatory response syndrome (SIRS). Severe SIRS is commonly accompanied by multiple organ dysfunction syndrome, ultimately resulting in multiple organ failure $[6,7]$.

Parecoxib, a non-steroidal anti-inflammatory drug (NSAID), is a second generation of cyclooxygenase-2 (COX-2) inhibitor. It mediates antipyretic, anti-inflammatory and analgesic effects by selectively inhibiting COX-2 activity and the production of prostaglandins and thromboxane A2 (TXA2) [8]. In this study, patients undergoing thoracoscopic surgery were enrolled to evaluate the ameliorative effects of parecoxib, in combination with ultrasound-guided paravertebral block (UGPB) against pain, stress response, and inflammation after thoracoscopic surgery. These studies may provide a basis for the use of parecoxib in post-operative analgesia.

\section{EXPERIMENTAL}

\section{Subjects}

In the present study, thoracoscopic surgery patients were selected from March 2016 to March 2017 in the Department of Anesthesiology of Yantaishan Hospital (Yantai, China). All patients underwent general anesthesia combined with UGPB. The experimental protocols were carried out in accordance with the declaration of Helsinki [9], and approved by the Ethics Committee of Shandong Provincial Hospital Affiliated to Shandong University (Ji'nan, China) (no. 2015-hu35-1).

\section{Experimental protocol}

All patients underwent the perioperative management model according to the concept of enhanced recovery after surgery (ERAS): no drinking $2 \mathrm{~h}$ before surgery, no urine catheter, no pre-operative drug administration, and standard preoperative preparation and anesthesia process.

UGPB was performed before general anesthesia by administering $20 \mathrm{~mL}$ ropivacaine $(0.5 \%)$ between the T4 and T8 vertebrae. The anesthesia plane was determined 10 min after blocking. All enrolled patients were randomly divided into two groups: placebo (control group, $\mathrm{n}$ $=20$ ) and parecoxib (experimental, $n=20$ ). Before operation, patients were intravenously administered parecoxib (40 mg) or placebo (normal saline), which was administered once again $24 \mathrm{~h}$ after surgery.

\section{Determination of ET, TXA2, IL- 6 and TNF- $\alpha$ in arterial blood}

Arterial blood samples were collected before operation (T0), 24h after operation (T1) and $48 \mathrm{~h}$ after operation (T2). The blood was centrifuged at $3500 \mathrm{rpm}$ for $15 \mathrm{~min}$ at $4^{\circ} \mathrm{C}$. The plasma was then collected and stored at $-80^{\circ} \mathrm{C}$. The levels of endotoxin (ET), thromboxane A2 (TXA2), interleukin 6 (IL-6), and tumor necrosis factor alpha (TNF- $\alpha$ ) in arterial blood were measured using commercial ELISA kits following the manufacturer's instructions. The opioid (fentanyl) dosage, infusion volume, blood loss, operation time, visual analog scale (VAS) score at 24 and $48 \mathrm{~h}$, and length of post-operative hospital stay were also recorded.

\section{Statistical analysis}

All data were expressed as mean tstandard deviation (SD), and analyzed by SPSS software (SPSS for Windows 19.0, SPSS Inc., USA). Oneway analysis of variance (ANOVA, Dunnett's multiple comparisons test) was used for analyzing differences between groups, and $p<$ 0.05 was considered significant.

\section{RESULTS}

\section{Demographic profile of patients}

A total of 40 patients were enrolled in the study. There were no significant differences in age, sex, height, body weight, fentanyl dosage, surgery time, blood loss and infusion volume between the parecoxib and control groups (Table 1).

\section{Post-operative VAS score and hospital stay}

As shown in Figure 1, the VAS scores of patients in the parecoxib group were significantly lower than the control group at 24 and $48 \mathrm{~h}$ after surgery $(p<0.05)$. In addition, the hospitalization period of the parecoxib treatment group was significantly shorter than the control group $(p<$ 0.05) (Table 1). 
Table 1: Demographic characteristics of thoracoscopic surgery patients

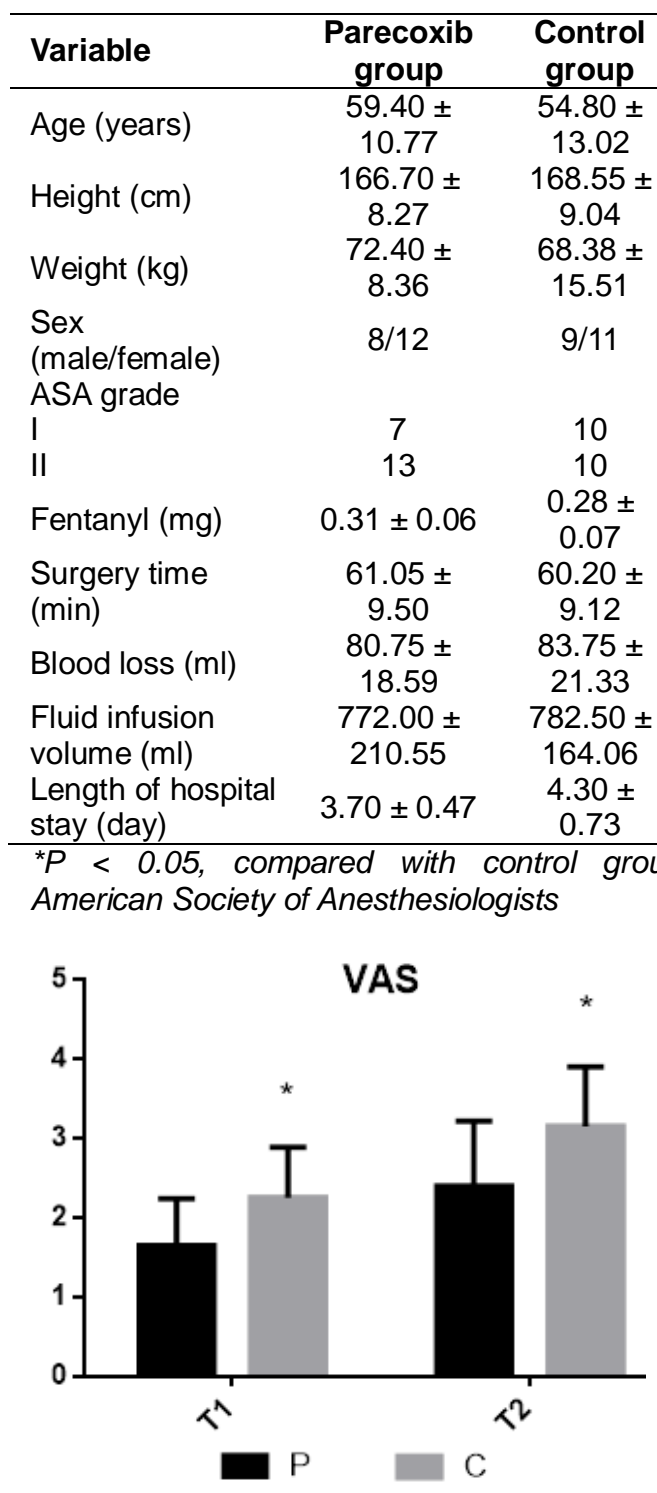

Figure 1: VAS scores of the patients. $\mathrm{P}$, parecoxib group; C, control group; T1, $24 \mathrm{~h}$ after surgery; T2, 48 $\mathrm{h}$ after surgery. ${ }^{*} p<0.05$, compared with control group

\section{Plasma levels of ET, TXA2, IL- 6 and TNF- $\alpha$}

There were no significant differences in plasma levels of ET (Figure 2), TXA2 (Figure 3), IL-6 (Figure 4) and TNF- $\alpha$ (Figure 5) between the parecoxib group and the control group before surgery $(p>0.05)$. Interestingly, plasma levels of ET, TXA2, IL-6, and TNF- $\alpha$ in the parecoxib group reduced compared to the control group 24 $\mathrm{h}$ after surgery $(p<0.05)$. However, no significant difference was observed between the two groups $48 \mathrm{~h}$ after surgery $(p>0.05)$.

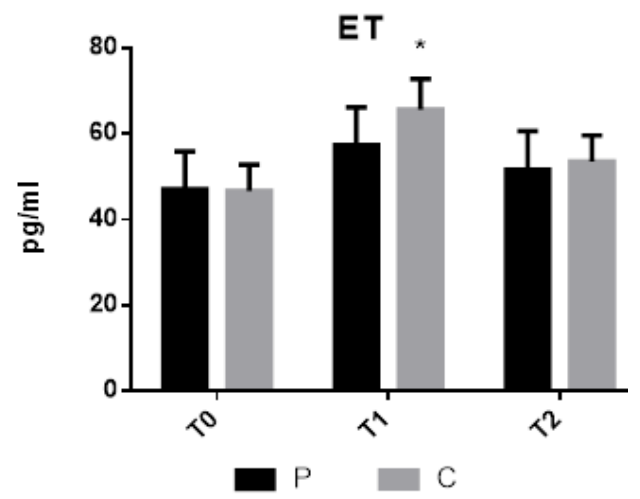

Figure 2: Endotoxin plasma levels of the patients. ET, endotoxin; P: parecoxib group; C: control group; T0, before surgery; T1, $24 \mathrm{~h}$ after surgery; T2, $48 \mathrm{~h}$ after surgery. ${ }^{*} p<0.05$, compared with control group.

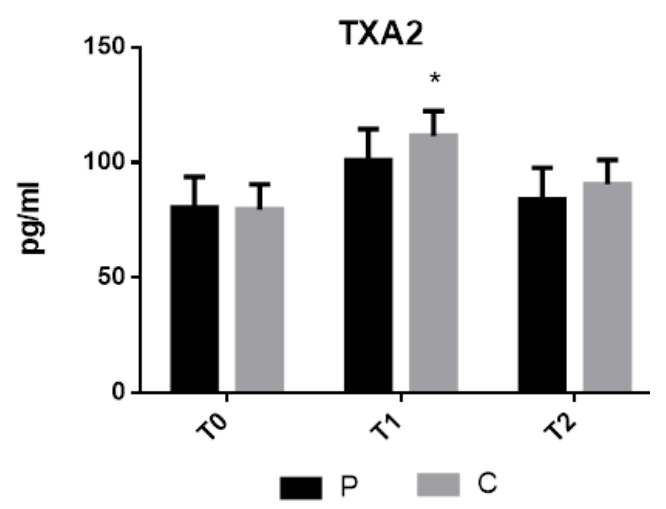

Figure 3: Thromboxane A2 plasma levels of the patients. TXA2, thromboxane A2; $\mathrm{P}$, parecoxib group; $\mathrm{C}$, control group; T0, before surgery; T1, $24 \mathrm{~h}$ after surgery; T2, $48 \mathrm{~h}$ after surgery; * $p<0.05$, compared with control group

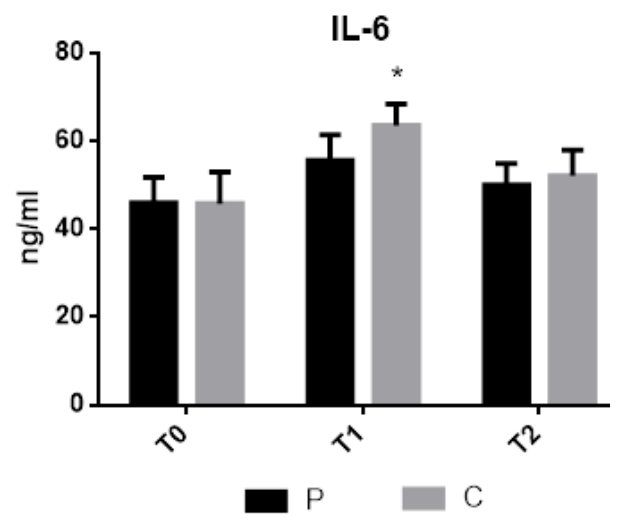

Figure 4: Interleukin 6 plasma levels of the patients. IL-6, interleukin 6; $\mathrm{P}$, parecoxib group; $\mathrm{C}$, control group; T0, before surgery; T1, 24 h after surgery; T2, $48 \mathrm{~h}$ after surgery. ${ }^{*} p<0.05$, compared with control group. 


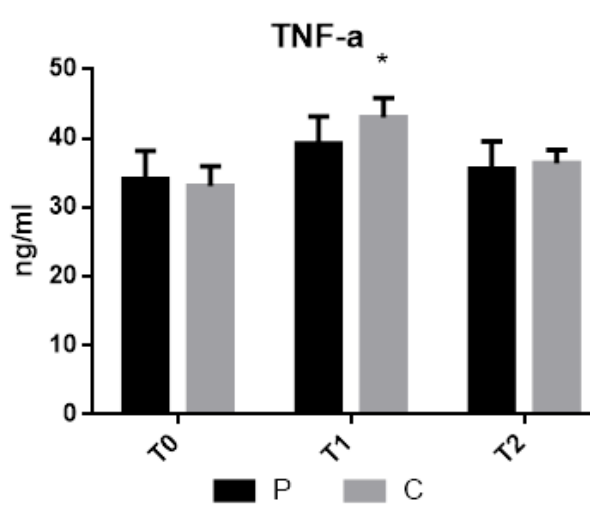

Figure 5: TNF- a plasma levels of the patients. $P$, parecoxib group; C, control group; T0, before surgery; $\mathrm{T} 1,24 \mathrm{~h}$ after surgery; T2, $48 \mathrm{~h}$ after surgery. ${ }^{*} p<$ 0.05 , compared with control group

\section{DISCUSSION}

Lung cancer is the most common cancer in the world, and its incidence is increasing. Lobectomy is commonly used for the treatment of lung cancer. However, the surgery often affects patients' postoperative quality of life and easily leads to chronic pain [10]. Thoracic surgery patients usually have deep breathing difficulty and cough expectoration due to the severe pain, which can also lead to respiratory secretions retention, small airway blocking, atelectasis, hypothyroidism and inflammatory responses. In 2001, researchers developed the concept of ERAS for reducing the average length of postoperative hospital stays and improving the quality of patients after surgery $[12,13]$.

Post-operative rehabilitation quality is influenced by many factors, including surgical procedure and time, stress and inflammatory response, intra-operative anesthesia quality, post-operative analgesic quality, and postoperative complications [11]. Thus, the levels of inflammatory cytokines in the plasma, opioid drug dosage, infusion volume, blood loss, surgery time, VAS score, and post-operative hospital stay were recorded in this study to evaluate the efficacy of the therapeutic method.

It is well known that NSAIDs possess favorable anti-inflammatory and analgesic activities. However, traditional non-selective NSAIDs often have side-effects, such as nausea, vomiting and stomach bleeding. [14]. Parecoxib is a novel selective NSAID that targeted COX-2 and, has fewer side-effects than traditional non-selective NSAIDs. Parecoxib analgesia can prevent intraoperative nociceptive stimulation, caused by central sensitization and pain up-regulation, to block or reduce the occurrence of postoperative pain. TNF- $\alpha$ and IL- 6 are well-known important pro-inflammatory cytokines. TNF- $\alpha$ is a key cytokine in the initiation of systemic inflammatory response, and IL- 6 is a downstream cytokines in the inflammatory process.

Additionally, these two pro-inflammatory cytokines are involved in activation of the inflammatory reaction cascade in sepsis and play important roles in the pathogenesis of SIRS [15]. ET induces the activation of endothelial cells in the stress response and is harmful to some organs and tissues. TXA ${ }_{2}$ is also produced in stress response. Thus, ET and $\mathrm{TXA}_{2}$ reflect the level of stress response $[16,17]$. In the present study, the treatment of parecoxib, combined with UGPB, significantly decreased the plasma levels of ET, TXA2, IL-6, and TNF- $\alpha$, indicating that this method alleviated the stress and inflammatory response induced by thoracoscopic surgery.

\section{CONCLUSION}

Parecoxib, combined with UGPB, effectively relieves the pain, stress responses, and inflammatory response in patients after thoracoscopic surgery. This treatment would be beneficial for improving the postoperative quality of life of lung cancer and other thoracoscopic surgery patients.

\section{DECLARATIONS}

\section{Conflict of Interest}

No conflict of interest associated with this work.

\section{Contribution of Authors}

We declare that this work was done by the authors named in this article and all liabilities pertaining to claims relating to the content of this article will be borne by the authors. Furthermore, Zhijian Fu conceived and designed the study, Xiaoning Cui collected and analyzed the data, and Xuejie $\mathrm{Li}$ and Shouhong Zhang wrote the manuscript. All authors have read and approved the manuscript for publication.

\section{REFERENCES}

1. Harada A, Jogie-Brahim $S$, Oh $Y$. Tobacco specific carcinogen 4-(methylnitrosamino)-1-(3-pyridyl)-1butanone suppresses a newly identified anti-tumor IGFBP-3/IGFBP-3R system in lung cancer cells. Lung Cancer 2013; 80(3): 270-277.

2. Tezuka Y, Endo S, Matsui A, Sato A, Saito K, Semba K, Takahashi M, Murakami T. Potential anti-tumor effect of IFN- 22 (IL-28A) against human lung cancer cells. Lung Cancer 2012; 78(3):185-192.

Trop J Pharm Res, June 2018; 17(6): 1174 
3. Li XT, He ML, Zhou $Z Y$, Jiang $Y$, Cheng $L$. The antitumor activity of PNA modified vinblastine cationic liposomes on Lewis lung tumor cells: In vitro and in vivo evaluation. Int J Pharm. 2015; 487(1-2): 223-233.

4. Argoff CE. Recent management advances in acute postoperative pain. Pain Pract 2014; 14(5): 477-487.

5. Baue AE, Durham R, Faist E. Systemic inflammatory response syndrome (SIRS), multiple organ dysfunction syndrome (MODS), multiple organ failure (MOF): are we winning the battle? Shock 1998; 10(2): 79-89.

6. Wei H, Choudhary W, He R, Ouyang C, Cheng Z, Liu D, Lu F, Liu X. Colorectal carcinoma masked by systemic inflammatory response syndrome: $A$ case report. Oncol Lett 2017; 14(4): 4906-4910.

7. Wesolek JL, McNorton K, Delgado G Jr, Giuliano CA. Effect of vancomycin initial dosing on time to systemic inflammatory response syndrome resolution in patients with methicillin-resistant Staphylococcus aureus bacteremia. J Chemother 2017, 25: 1-6.

8. Essex MN, Xu H, Parsons B, Xie L, Li C. Parecoxib relieves pain and has an opioid-sparing effect following major gastrointestinal surgery. Int J Gen Med 2017; 10 : 319-327.

9. World Medical Organization. Declaration of Helsinki. Brit Med J 1996; 313(7070): 1448-1449.

10. Appel S, Goldstein J, Perelman M, Rabin T, Urban D, Onn A, Shulimzon TR, Weiss I, Lieberman S, Marom EM. Neo-adjuvant Chemo-Radiation to 60 Gray Followed by Surgery for Locally Advanced Non-Small Cell Lung Cancer Patients: Evaluation of Trimodality Strategy. Isr Med Assoc J 2017; 19(10): 614-619.

11. Jiang $Y$, Liu T. Effect of operating room care combined with home care for the postoperative rehabilitation and prognosis of gastric cancer patients with low PTEN gene expression. Oncol Lett 2017; 14(2): 2119-2124.

12. Bray MS, Appel AL, Kallies KJ, Borgert AJ, Zinnel BA, Shapiro SB. Implementation of an Enhanced Recovery After Surgery Program for Colorectal Surgery at a Community Teaching Hospital. WMJ 2017; 116(1):2226.

13. Pecorelli $N$, Fiore JF Jr, Kaneva $P$, Somasundram A, Charlebois $P$, Liberman AS, Stein BL, Carli F, Feldman LS. An app for patient education and self-audit within an enhanced recovery program for bowel surgery: a pilot study assessing validity and usability. Surg Endosc 2017. doi: 10.1007/s00464-017-5920-3.

14. Tsagareli MG, Nozadze I, Tsiklauri N, Gurtskaia G. Nonsteroidal anti-inflammatory drugs attenuate agonistevoked activation of transient receptor potential channels. Biomed Pharmacother 2017; 97: 745-751.

15. Jo AR, Han HS, Seo S, Shin JS, Lee JY, Kim HJ, Lee $K T$. Inhibitory effect of moschamine isolated from Carthamus tinctorius on LPS-induced inflammatory mediators via AP-1 and STAT1/3 inactivation in RAW 264.7 macrophages. Bioorg Med Chem Lett 2017; pii: S0960-894X (17)31020-X.

16. Brooks AC, Menzies-Gow NJ, Wheeler-Jones C, Bailey SR, Cunningham FM, Elliott J. Endotoxin-induced activation of equine platelets: evidence for direct activation of p38 MAPK pathways and vasoactive mediator production. Inflamm Res 2007; 56(4): 154-161.

17. Bezugla Y, Kolada A, Kamionka S, Bernard B, Scheibe $R$, Dieter P. COX-1 and COX-2 contribute differentially to the LPS-induced release of PGE2 and TXA2 in liver macrophages. Prostaglandins Other Lipid Mediat 2006; 79(1-2): 93-100. 